\title{
Immunohistochemical detection of cdc2 is useful in predicting survival in patients with mantle cell lymphoma
}

\author{
David Hui ${ }^{1}$, Tony Reiman ${ }^{1,2}$, John Hanson ${ }^{2}$, Rick Linford ${ }^{2}$, Winson Wong ${ }^{3}$, Andrew Belch ${ }^{1,2}$ \\ and Raymond Lai ${ }^{2,3}$ \\ ${ }^{1}$ Department of Medicine, Faculty of Medicine and Dentistry, University of Alberta, Edmonton, Alberta, \\ Canada; ${ }^{2}$ Cross Cancer Institute, Edmonton, Alberta, Canada; and ${ }^{3}$ Department of Laboratory Medicine and \\ Pathology, Faculty of Medicine and Dentistry, University of Alberta, Edmonton, Alberta, Canada
}

\begin{abstract}
Recent cDNA microarray studies have reported the prognostic value of several genes in mantle cell lymphoma patients. We aimed to validate the prognostic significance of three of these genes: $\alpha-t u b u l i n, c d c 2$, and CENP-F. The protein expression of $\alpha$-tubulin, cdc2, and CENP-F was assessed using immunohistochemistry. Their immunoreactivity in 48 formalin-fixed/paraffin-embedded mantle cell lymphoma tumors was determined by estimating the percentage of positive cells. These results were correlated with the expression of proliferation marker Ki67 and survival. Of these 48 mantle cell lymphoma patients, 41 were men and seven were women. The median age at time of diagnosis was 64.5 years, and the overall median survival was 40 months. In benign lymph nodes, the expression of cdc2 and $\alpha$-tubulin was restricted to the germinal centers; mantle zones were negative. Expression of CENP-F was more uniformly distributed. In mantle cell lymphoma, Ki67 significantly correlated with all three markers $(P<0.05$, Spearman), but only Ki67 ( $>50 \%)$ and cdc2 ( $>25 \%)$ significantly correlated with shorter survival $(P<0.0006$, Spearman). Of several clinical parameters examined, international prognostic index of $\geq 2$ correlated with worse clinical outcome, and high clinical stage (ie 4 vs $\leq 3$ ) showed a trend for shorter survival. The prognostic significance of cdc2 and Ki67 was independent of international prognostic index and clinical stage. We have validated the prognostic value of cdc2, and confirmed that of Ki67, in a cohort of mantle cell lymphoma patients. Immunohistochemical detection of cdc2 and Ki67 may be a useful and simple method in evaluating the prognosis of mantle cell lymphoma patients.

Modern Pathology (2005) 18, 1223-1231. doi:10.1038/modpathol.3800409; published online 3 June 2005
\end{abstract}

Keywords: mantle cell lymphoma; prognosis; cdc2; Ki67; immunohistochemistry

Mantle cell lymphoma is an aggressive nonHodgkin's lymphoma of mature B-cell immunophenotype, characterized by the chromosomal translocation $\mathrm{t}(11 ; 14)$ and overexpression of cyclin D1. ${ }^{1}$ The median survival of mantle cell lymphoma patients is roughly 3 years, but it has been recognized that the length of survival is relatively heterogeneous. ${ }^{2,3}$ Various studies have been performed in evaluating the prognostic importance of a number of clinical, pathologic, and biologic markers. For the pathologic parameters, blastoid and

Correspondence: Dr R Lai, MD, PhD, Department of Laboratory Medicine and Pathology, Faculty of Medicine and Dentistry, University of Alberta and Cross Cancer Institute, 4B1 Walter MacKenzie Health Sciences Center, 8440 - 112 Street, Edmonton, Alberta, Canada T6G 2B7.

E-mail: Raymondlai@cha.ab.ca

Received 8 November 2004; revised and accepted 28 January 2005; published online 3 June 2005 mantle zone mantle cell lymphoma have been reported to correlate with shorter and longer survival respectively, ${ }^{4,5}$ although the frequencies of these mantle cell lymphoma variants are relatively low. For the clinical parameters, patient age, the presence of splenomegaly or anemia, but not international prognostic index (IPI) and Ann Arbor clinical stage, were found to be associated with survival in one study. ${ }^{2}$ Nevertheless, IPI and its determinants, including bone marrow involvement, performance status, advanced stage disease, were found to predict prognosis in another study. ${ }^{3}$ For the biologic markers, high proportion of cells positive for Ki67 has been found to correlate with worse clinical outcome..$^{4,6,7}$ In addition, genetic alterations in $\mathrm{p} 53^{8-10}$ and loss of $\mathrm{p} 27^{11}$ are associated with worse clinical outcome. While these biologic markers have been shown to be useful in predicting survival, these assays have not be carried out in most routine diagnostic pathology laboratories. 
The pathogenesis of mantle cell lymphoma is incompletely understood. While cyclin D1 overexpression is believed to contribute to the pathogenesis of this disease, this genetic defect alone is not sufficient in inducing tumorigenesis in a transgenic mouse model. ${ }^{12}$ More recent studies employing genome-scale expression profiles showed that mantle cell lymphoma cells have deregulation in multiple cellular pathways and processes, involving apoptosis, cell cycle progression, signal transduction, and cell structure. ${ }^{13,14}$ These data not only shed light into the pathogenesis of mantle cell lymphoma, but also provide an opportunity to assess the prognostic value of the expression of various genes in these tumors. Rosenwald et al identified 48 mantle cell lymphoma signature genes that are significantly correlated with survival of mantle cell lymphoma patients. Most (28 of 48) of these genes are associated with cellular proliferation, including genes required for DNA replication, cell cycle progression, and the metabolic demands for DNA replication. The authors also constructed a model taking into account the expression level of four signature genes, $\alpha$-tubulin, cdc2, CENP-F, ASPM, and they found that this model predicted survival with high statistical significance.

Since antibodies are commercially available for $\alpha$ tubulin, cdc2, and CENP-F, we tested the prognostic value of these three markers using standard immunohistochemistry and formalin-fixed/paraffinembedded tissues from 48 mantle cell lymphoma tumors. The protein expression level of each of these three markers was correlated with the Ki67 expression level (ie proliferation index) and statistical analysis was performed to assess the prognostic value of these markers. Validation of these markers using immunohistochemistry may allow prognostic assessment for mantle cell lymphoma patients to be readily performed in most diagnostic pathology laboratories.

\section{Materials and methods}

\section{Patient Samples}

A total 48 mantle cell lymphoma patients, who were initially diagnosed at the Cross Cancer Institute between 1993 and 2004, were identified from the file at the Department of Laboratory Medicine and Pathology, Cross Cancer Institute. Formalin-fixed/ paraffin tissue blocks were available for all of these 48 cases. The use of these human tissue samples was approved by the Institutional Ethics Committee. The diagnosis of all 48 mantle cell lymphoma was reviewed and confirmed using criteria described in the WHO Classification scheme for hematologic malignancies. ${ }^{1}$ All cases were positive for CD5 and/or CD43 and cyclin D1, and negative for CD23. Two cases were of blastoid variant and none showed a predominantly mantle zone growth pattern. Treatment for each mantle cell lymphoma patient was determined during our weekly lymphoma rounds for new patients, based on our institutional protocol.

\section{Tissues and Immunohistochemistry}

Mantle cell lymphoma tissue microarrays containing tissue cores from each mantle cell lymphoma tumor and two benign lymph nodes were constructed. Monoclonal antibodies reactive with $\alpha$ tubulin and cdc2 were purchased from Santa Cruz Biotechnology (Santa Cruz, CA, USA). Polyclonal antibody to CENP-F was obtained from Novus Biologicals (Littleton, CO, USA). Immunohistochemical staining was performed using standard techniques. Briefly, tissue sections of $4 \mu \mathrm{M}$ thickness from the mantle cell lymphoma tissue microarrays were deparaffinized and rehydrated with graded alcohol. Heat-induced epitope retrieval was performed using Tris buffer (pH 9.9; Dako, Mississauga, Ontario, Canada) and a rapid microwave histoprocessor (RHS, Milestone, Bergamo, Italy). Endogenous peroxidase activity was blocked by incubating samples in $3 \% \mathrm{H}_{2} \mathrm{O}_{2}$ for $10 \mathrm{~min}$, followed by a wash with water and then phosphate-buffered saline (PBS; pH 7.2). Tissue sections were then incubated with anti- $\alpha$-tubulin antibody $(1: 400)$, anti-cdc2 antibody (1:100), or anti-CENP-F antibody (1:200) overnight in a humidified chamber at $4^{\circ} \mathrm{C}$. After three washes with PBS, tissue sections were incubated with anti-mouse or anti-rabbit IgG and peroxidase (EnVision, Dako) for $30 \mathrm{~min}$ at room temperature. After three washes with PBS, tissue sections were incubated with 3,3'-diaminobenzidine $/ \mathrm{H}_{2} \mathrm{O}_{2}$ (Dako) for color development and hematoxylin was used as a counterstain. Duplicated experiments were performed. For Ki67 immunostaining, monoclonal antibody was purchased from Immunotech (Westbrook, MN, USA; 1:120), and the method was similar to that for the other three antibodies, except that the incubation for the primary antibody was $30 \mathrm{~min}$ at room temperature.

For each case, a score of $0-4$ was given by two independent observers based on the estimate of the percentage of cells showing definitive staining, regardless of the staining intensity $(0=$ no stain, $1=$ less than $10 \%$ of cells, $2=10-25 \%, 3=26-50 \%$, $4=>50 \%$ ). Evaluation of immunostaining was performed without knowing the clinical outcome and the other staining results. The final score for each marker was the average of the scores obtained by the two observers (DH, RL). Cases with major discrepancy in scoring (ie $>1$ ) were reviewed by both observers on a multiheaded microscope.

\section{Statistical Analysis}

Logrank test was utilized for univariate survival comparisons, unstratified and stratified adjustment for stage, between different groups, and Kaplan- 
Meier survival estimates and median survival times were computed. Relative risk is the ratio of two proportions of patients showing disease progression and methods of calculation have been described. ${ }^{15}$ Two-sided tests were used in the statistical analysis and were performed using SAS software (version 8.1, SAS Institute, Cary, NC, USA).

\section{Results}

\section{Clinical and Demographic Characteristics}

The clinical and demographic data of the 48 mantle cell lymphoma patients are summarized in Table 1. There were 41 men and seven women $(\mathrm{M}: \mathrm{F}=6.7: 1)$. The median age at the time of diagnosis was 64.5 years, with $35(73 \%)$ patients being 60 years of age or older. The overall median survival was 40 months (Figure 1). In all, $29(60 \%)$ died during the followup. IPI was retrievable on 30 patients; seven (23\%) patients had an IPI of 0 to 1 and $23(77 \%)$ had an IPI of 2 to 5. Serum lactate dehydrogenase (LDH) level was elevated in six of $31(19 \%)$ patients. Most (31/ 47, 66\%) patients were in Ann Arbor stage IV at time of diagnosis, and most $(33 / 42,79 \%)$ had a performance status of 0 or 1 . With regard to treatment, 21 patients received CHOP (cyclophosphamide, doxorubicin, vincristine, prednisone)-based therapy, 11 received chlorambucil-based therapy, and 10 re-

Table 1 Demographic and clinical characteristics of 48 mantle cell lymphoma patients

\begin{tabular}{|c|c|}
\hline \multicolumn{2}{|c|}{ Gender (48 patients ${ }^{\mathrm{a}}$ ) } \\
\hline Male & $41(85 \%)$ \\
\hline Female & $7(15 \%)$ \\
\hline \multicolumn{2}{|c|}{ Age (48 patients ${ }^{\mathrm{a}}$ ) } \\
\hline$<60$ & $13(27 \%)$ \\
\hline$\geq 60$ & $35(73 \%)$ \\
\hline \multicolumn{2}{|c|}{ International prognostic index (30 patients $\left.{ }^{\mathrm{a}}\right)$} \\
\hline $0-1$ & $7(23 \%)$ \\
\hline $2-3$ & $19(63 \%)$ \\
\hline $4-5$ & $4(13 \%)$ \\
\hline \multicolumn{2}{|c|}{ Lactate dehydrogenase (31 patients ${ }^{\mathrm{a}}$ ) } \\
\hline Normal & $25(81 \%)$ \\
\hline Elevated & $6(19 \%)$ \\
\hline \multicolumn{2}{|c|}{ Stage (47 patients ${ }^{\mathrm{a}}$ ) } \\
\hline I-III & $16(34 \%)$ \\
\hline IV & $31(66 \%)$ \\
\hline \multicolumn{2}{|c|}{ Performance status (42 patients ${ }^{\mathrm{a}}$ ) } \\
\hline 0,1 & $33(79 \%)$ \\
\hline $2,3,4$ & $9(21 \%)$ \\
\hline \multicolumn{2}{|c|}{ Extranodal sites ${ }^{\mathrm{b}}$ (45 patients ${ }^{\mathrm{a}}$ ) } \\
\hline 0,1 & $40(89 \%)$ \\
\hline$>1$ & $5(11 \%)$ \\
\hline
\end{tabular}

${ }^{\mathrm{a}}$ Indicates the number of patients with data available.

${ }^{\mathrm{b}}$ Extranodal sites peripheral blood and bone marrow involvement were considered together as one site.

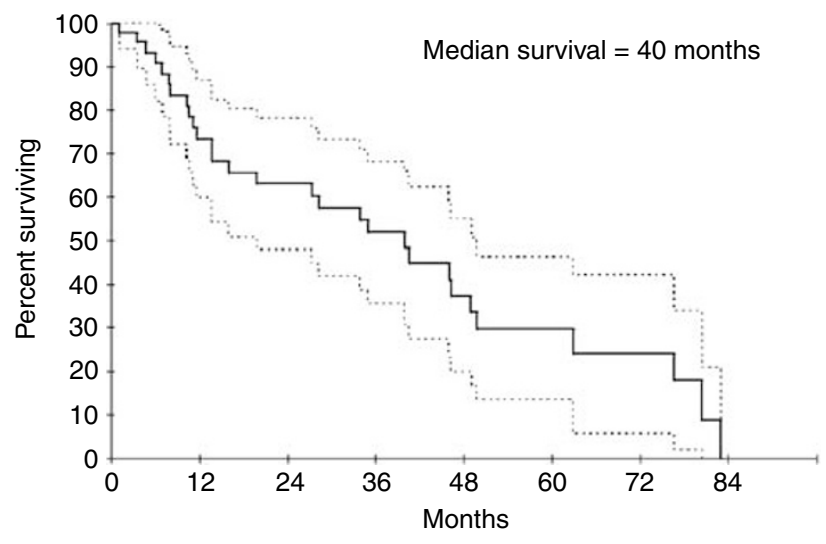

Figure 1 Kaplan-Meier survival curve of 48 mantle cell lymphoma patients.

ceived a variety of other treatments including proteasome inhibitor PS341 (two patients) and cyclin-dependent kinase modulator flavopiridol (three patients).

\section{Immunohistochemical Staining}

In reactive lymph nodes, $\alpha$-tubulin, cdc2, and Ki67 were largely confined to the germinal centers; mantle zones were negative (Figure 2a,b). CENP-F immunostaining was also found mostly in the germinal centers, although a relatively higher number of positive cells were found in the mantle zone as well as the interfollicular areas (Figure 2c). The immunostaining results of 48 mantle cell lymphoma tumors are summarized in Table 2 and illustrated in Figure $2 \mathrm{~d}-\mathrm{g}$. $\alpha$-Tubulin showed cytoplasmic staining, and most $(26 / 48,54 \%)$ mantle cell lymphoma tumors showed $>25 \%$ positive cells (ie a score of 3 or 4). In contrast, most mantle cell lymphoma tumors had relatively small numbers of positive cells for cdc2 and CENP-F, with only $8 / 48(16 \%)$ and $9 / 48(22 \%)$ mantle cell lymphoma tumors carrying $>25 \%$ positive cells for cdc2 and CENP-F, respectively. Roughly half (21/46, 46\%) of mantle cell lymphoma cases had $>25 \%$ cells reactive with Ki67. The immunostaining of cdc2 was predominantly cytoplasmic, although a lesser extent of nuclear staining was also identified. The immunostaining patterns of CENP-F and Ki67 were nuclear.

We have assessed intra- and inter-observer variability in scoring the immunostaining for these markers. The Spearman correlation coefficients between the two sets of scores from one observer (RL) were $0.96,0.81$, and 0.83 , for $\alpha$-tubulin, cdc2, and CENP-F, respectively. The Spearman correlation coefficients between the two sets of scores obtained from two different observers (DH, RL) were 0.95, 0.83 , and 0.85 for $\alpha$-tubulin, cdc2, and CENP-F, respectively. 

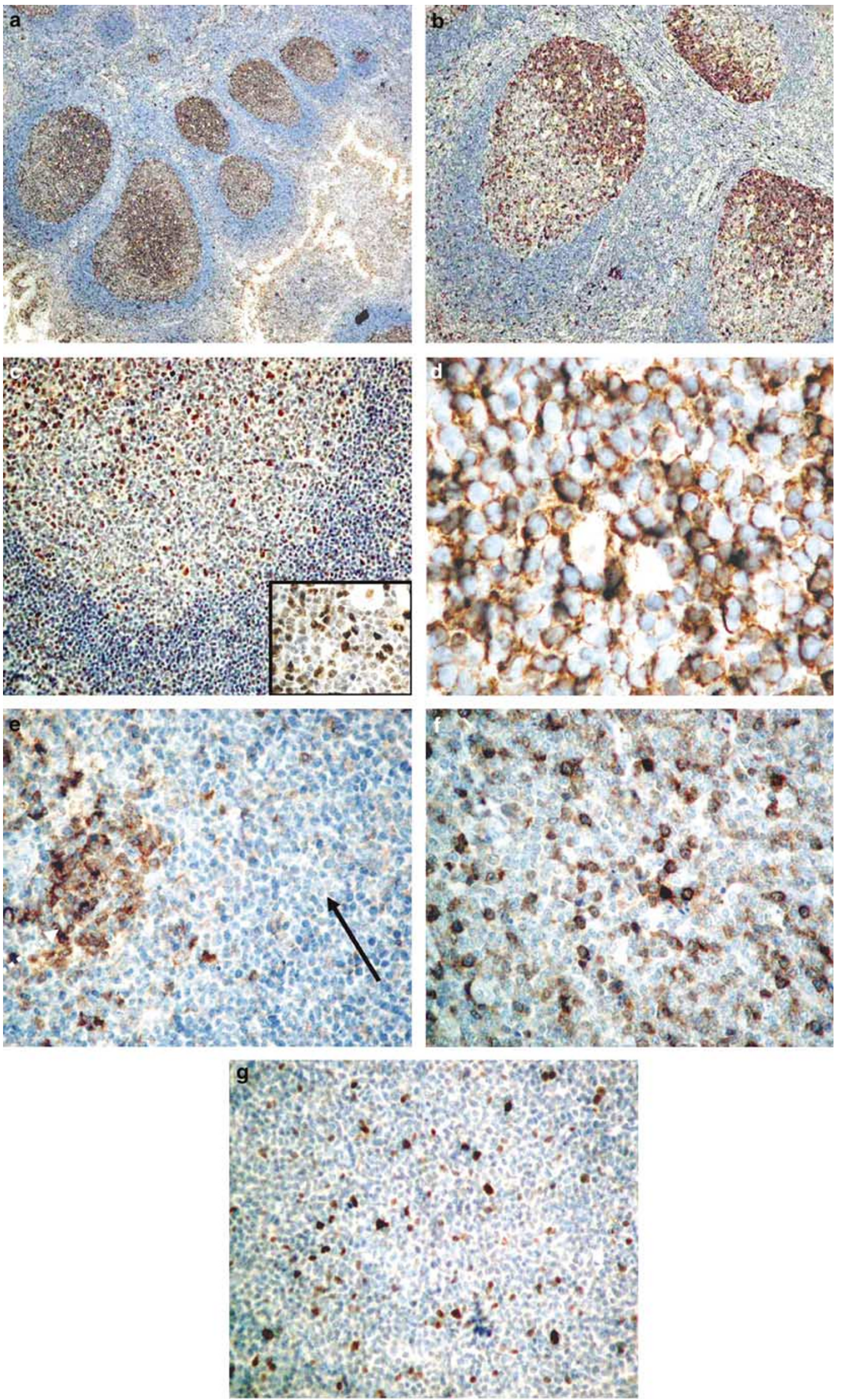
Table 2 Immunoreactivity for $\alpha$-tubulin, cdc2, CENP-F, and Ki67 in 48 mantle cell lymphoma tumors

\begin{tabular}{lcccc}
\hline $\begin{array}{l}\text { Immunostaining } \\
\text { scores }\end{array}$ & $\alpha$-Tubulin & cdc2 & CENP-F & Ki67 \\
\hline 0 & & & & \\
$0-1$ & $2(4 \%)$ & $4(8 \%)$ & $2(4 \%)$ & $2(4 \%)$ \\
$>1-2$ & $5(10 \%)$ & $19(40 \%)$ & $18(38 \%)$ & $4(9 \%)$ \\
$>2-3$ & $9(19 \%)$ & $17(35 \%)$ & $17(35 \%)$ & $19(41 \%)$ \\
$>3-4$ & $6(13 \%)$ & $5(10 \%)$ & $6(13 \%)$ & $11(24 \%)$ \\
Total cases evaluated & $26(54 \%)$ & $3(6 \%)$ & $5(10 \%)$ & $10(22 \%)$ \\
& 48 & 48 & 48 & 46
\end{tabular}

The numbers in the table indicate the number of cases positive for each scoring category.

\section{Correlation between Proliferation and $\alpha$-Tubulin, cdc2, and CENP-F}

To determine if the expression of $\alpha$-tubulin, cdc2, and CENP-F correlates with proliferation, we analyzed the correlation between Ki67 and these three markers. The Spearman correlation coefficients between Ki67 and $\alpha$-tubulin, cdc2, and CENP-F were $0.36 \quad(P<0.01), 0.64 \quad(P<0.0001)$, and 0.33 $(P<0.02)$, respectively.

\section{Correlation of Clinical Parameters with Mantle Cell Lymphoma Patient Survival}

Of the seven clinical parameters listed in Table 1, only a low IPI score (ie 0 or 1 ) showed a significant correlation with longer survival $(P=0.03$, logrank) (Figure 3). Statistical analysis using higher IPI scores as cutoffs did not reveal significant differences in the survival. Patients with a clinical stage of 1-3 also showed a trend for longer survival, but the association was not statistically significant $(P=0.15$, logrank).

\section{Prognostic Value of $\alpha$-Tubulin, cdc2, CENP-F, and Ki67 Expression}

High levels of $\alpha$-tubulin (ie $>2$ ) and CENP-F (ie $>2$ ) were not significantly correlated with overall survival, with relative risks being $0.88(P=0.74)$ and 1.05 $(P=0.91)$, respectively (Figure $4 \mathrm{a}$ and $\mathrm{b})$. Statistical analysis using different cutoffs did not reveal significant correlation with the survival for $\alpha$ tubulin and CENP-F. High levels of cdc2 expression $(>2)$ were strongly associated with short survival, with a relative risk of $4.31(P<0.0005)$ (Figure 4c). High level of Ki67 ( $>3$ ) was also found to be a useful prognostic marker (relative risk $=4.0, P<0.0006$ )

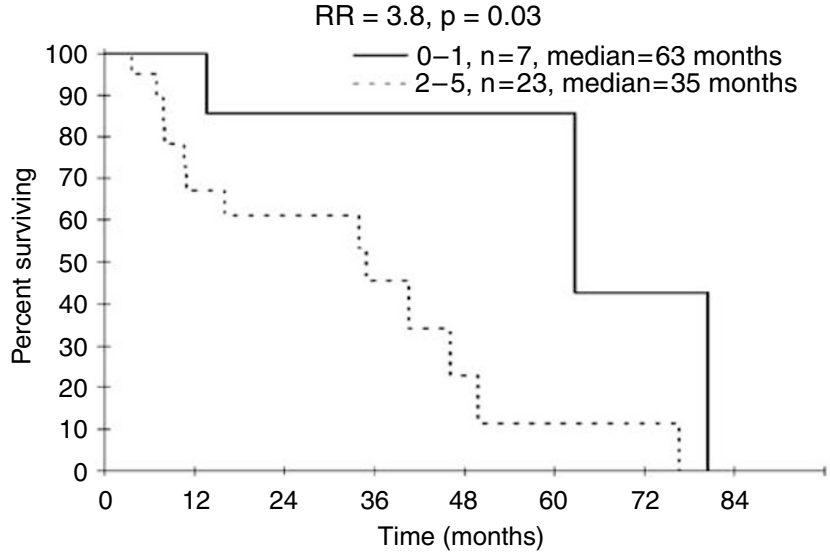

Figure 3 Kaplan-Meier survival curve of mantle cell lymphoma patients based on IPI (0-1 compared to 2-5).

(Figure 4d). A combined analysis correlating the sum of cdc2 and Ki67 with survival revealed a relative risk of $4.37(P<0.0002)$. A combined score of $\alpha$-tubulin, cdc2, and CENP-F yielded a relative risk of $3.84 \quad(P<0.007)$ for survival. Statistical analysis using different cutoffs for cdc2 and Ki67 did not reveal statistical significance.

Owing to the treatment heterogeneity in our patient group, we also assessed if there is any correlation between survival and high level of cdc2 $(>2)$ or Ki67 $(>3)$ in patients treated with CHOP therapy. In this patient group $(n=21)$, Ki67 remained to be significantly correlated with survival $(P=0.029$; relative risk $=4.73)$ while cdc2 only showed a trend $(P=0.14$; relative risk $=3.46)$. We also performed statistical analysis with the only two blastoid MCL cases removed; there was no significant changes to the $P$-values described.

Since we found that Ki67 and cdc2 are of prognostic value, we determined whether their prognostic significance is dependent of IPI (ie $<2$ vs $\geq 2$ ), the only clinical characteristics that we found to significantly correlate with overall survival. The data are summarized in Table 3. Ki67 remained to be prognostically significant after adjustment for IPI (relative risk $=3.63, P<0.0017$ ). Similarly, cdc2 remained to be prognostically significant after adjustment for IPI (relative risk $=3.20, P<0.0074$ ). Although the clinical stage (stage 1-3 vs 4) did not correlate significantly with survival, it appeared to show a trend (ie $P=0.15$ ). Thus, we also determined the prognostic significance of cdc2 and Ki67 after adjustment for clinical stage. As described in Table 3, both cdc2 and K67

Figure 2 Immunohistochemical staining of $\alpha$-tubulin, cdc2, CENP-F, and Ki67 in normal lymphoid tissue and mantle cell lymphoma tissues: (a) $\alpha$-tubulin in a tonsil; (b) cdc2 in a tonsil; (c) CENP-F in a tonsil, with insert showing nuclear staining; (d) $\alpha$-Tubulin expression in a case of mantle cell lymphoma; (e) low level of cdc2 expression in a case of mantle cell lymphoma, which was adjacent to a residual germinal center; (f) high level of cdc2 expression in a case of mantle cell lymphoma; (g) CENP-F expression in a case of mantle cell lymphoma. 

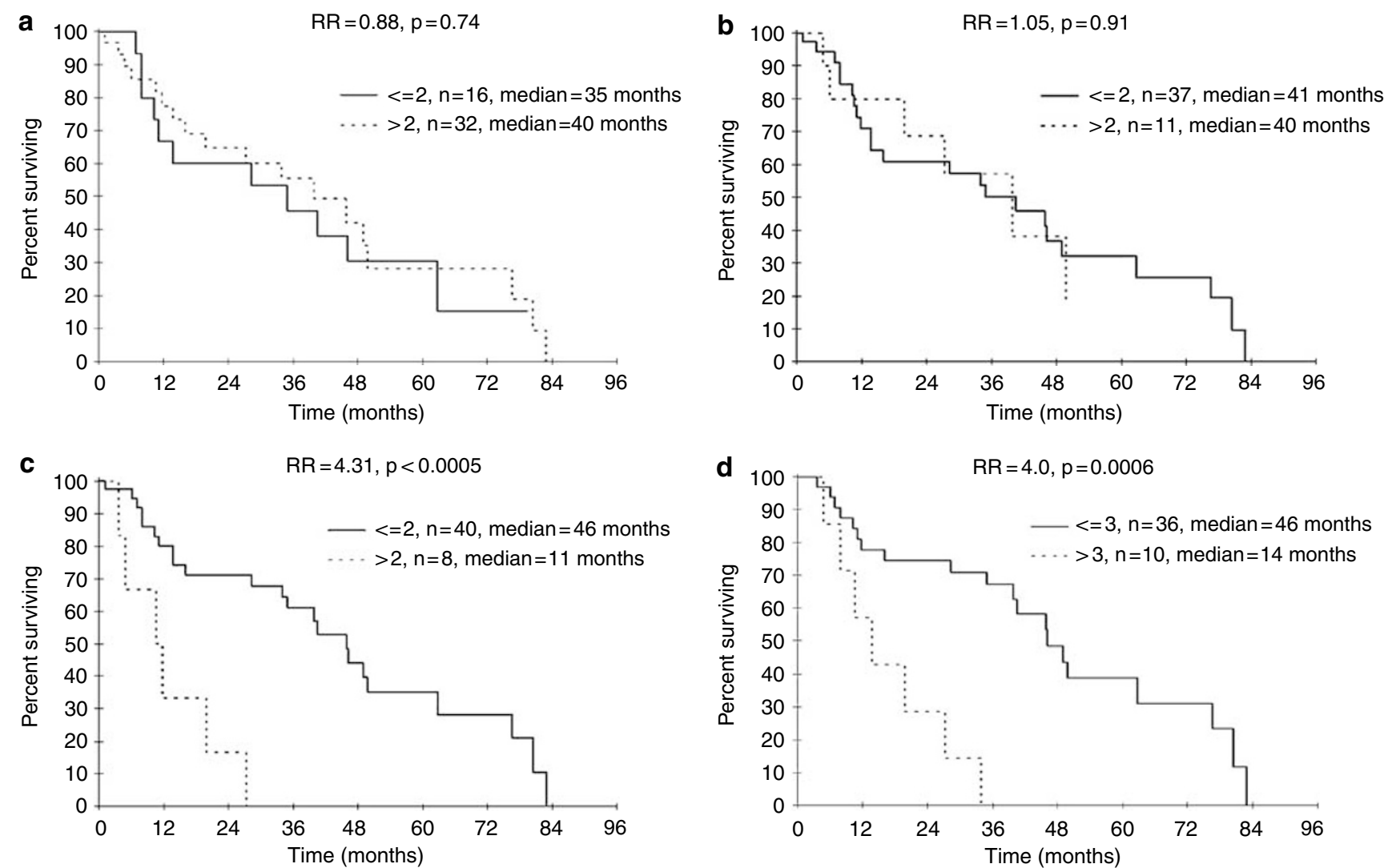

Figure 4 Kaplan-Meier survival curves of mantle cell lymphoma patients based on immunohistochemical staining of (a) $\alpha$-tubulin with a cutoff of 2, (b) CENP-F with a cutoff of 2, (c) cdc2 with a cutoff of 2, and (d) Ki67 with a cutoff of 3.

Table 3 Prognostic significance of cdc2, Ki67, adjusted for international prognostic index (IPI) and the Ann Arbor clinical stage in mantle cell lymphoma patients

\begin{tabular}{lccc}
\hline & Relative risk $(R R)$ & RR adjusted for IPI & RR adjusted for clinical stage \\
\hline Cdc2 $(>25 \%$ positive cells $)$ & $4.31(P<0.0005)$ & $3.20(P<0.0074)$ & $3.07(P<0.01)$ \\
Ki67 $(>50 \%$ positive cells $)$ & $4.00(P<0.0006)$ & $3.63(P<0.0017)$ & $2.78(P<0.016)$ \\
\hline
\end{tabular}

remained to be prognostically significant after adjustment for the clinical stage; the relative risk was $2.78(P<0.016)$ for $\mathrm{Ki67}$ and $3.07 \quad(P<0.01)$ for cdc2.

\section{Discussion}

The overall prognosis of mantle cell lymphoma patient is relatively poor, with a median survival of 3 years. Nevertheless, it has become clear that a subset of mantle cell lymphoma patients has relatively long survival. A number of previous studies have reported the prognostic values of various clinical, pathologic, and biologic markers for mantle cell lymphoma patients. ${ }^{2-11,16-18}$ Most mantle cell lymphoma patients present with a high clinical stage and widespread disease, and the prognostic value of IPI is controversial among studies. $^{2,4}$ Some biologic markers are promising, and these markers include $p 53$ mutations and loss of $p 27$, which have been found to correlate with worse clinical outcome in some studies. ${ }^{8-11}$

Several recent studies employing cDNA microarray techniques to study mantle cell lymphoma tumors have yielded a large amount of data related to genes that are upregulated or downregulated in mantle cell lymphoma compared to non-neoplastic B cells or other B-cell lymphoma types. ${ }^{13,14,19}$ Many of these genes are implicated in cellular proliferation and apoptosis. In addition to providing valuable insights into the pathogenesis of mantle cell lymphoma, the expression levels of some of these genes, as measured by quantitative PCR methods, 
have been shown to correlate with survival of mantle cell lymphoma patients. Martinez et al identified two clusters consisting of 26 genes that independently predict survival. Rosenwald et al also identified a number of genes that are predictive of short survival in mantle cell lymphoma patients. Interestingly, there was no overlap between the genes identified between these two studies. These discrepancies could be explained by the differences in the type of oligonucleotide microarrays and in the statistical methods for analyzing their data. Furthermore, Martinez et al compared mantle cell lymphoma tumors with normal tonsillar mantle zone B cells whereas Rosenwald et al compared mantle cell lymphoma tumors with other B-cell lymphomas. While quantitative RT-PCR was employed to correlate gene expression and survival, neither of these two studies assessed the protein expression of these genes. The main purpose of this study is to determine the prognostic utilities of three markers identified by Rosenwald et al, for which antibodies are commercially available. We aimed to establish a relatively feasible and technically simple method to provide prognostic information for mantle cell lymphoma patients.

We found that the three antibodies against $\alpha$ tubulin, cdc2, and CENP-F can be readily applied to routinely fixed/paraffin-embedded tissues with the use of standard antigen retrieval and immunohistochemistry. The staining pattern of $\alpha$-tubulin and CENP-F were in keeping with their expected subcellular localization, with $\alpha$-tubulin being in the cytoplasm and CENP-F being nuclear. For cdc2, we found that the staining pattern of cdc2 was predominantly cytoplasmic and partially nuclear. Similar staining pattern has been reported in epithelial cells of the oral cavity. ${ }^{20}$

All three antibodies against $\alpha$-tubulin, cdc2, and CENP-F highlighted the germinal centers in reactive lymph nodes, indicating that they may be related to cellular proliferation. In keeping with this concept, cdc2 (cyclin dependent kinase-1; cdk1) is a member of the family of cyclin-dependent kinases, which plays a critical role in regulating cell cycle progression. In one recent study, cdc2 expression was detectable in 5\% of primary thyroid lymphoma and its expression correlated with high Ki67 labeling and the grade of the tumor. ${ }^{21}$ In another study, expression of cdc2 was found to correlate with prognosis in a cohort of patients with B-cell nonHodgkin's lymphoma. ${ }^{22} \alpha$-Tubulin is a structural protein involved in the assembly of the mitotic spindles, and it is expected to increase in expression with increased mitotic activity. CENP-F is a member of the CENP centromere protein family, which is involved in the formation of the centromere and the organization/function of the kinetochore during mitosis. $^{23,24}$ CENP-F, also known as mitosin, has been found to correlate with tumor proliferation in various human neoplasms ${ }^{25,26}$ and survival in one study. ${ }^{27}$
We found that all three markers correlated with Ki67, further confirming the concept that they are associated with proliferation. While the idea that tumor proliferation is associated with worse prognosis is relatively well established in mantle cell lymphoma, ${ }^{4,6,15,17}$ the specific molecules involved in tumorigenesis have been elusive. Most recently, topoisomerase IIa, a key enzyme involved in DNA replication, has been found to be useful for survival prediction in mantle cell lymphoma. ${ }^{7,16}$ We report here the importance of cdc2 as both a proliferation indicator and prognostic marker. Indeed, cdc2 could play a key role in the pathogenesis of mantle cell lymphoma. Mantle cell lymphoma is characterized by an overexpression of cyclin D1, leading to dysregulation at the G1 checkpoint and driving cells into $\mathrm{S}$ phase. High levels of cdc2 expression would promote these tumor cells to progress further pass the G2 checkpoint. The loss of these two key cell cycle regulatory steps could provide an explanation for rapid tumor progression and shortened patient survival. Further supporting this double-hit hypothesis is that p53 plays an important regulatory role at both of these key transition points, and mutation in p53 has been associated with poorer survival in mantle cell lymphoma patients. ${ }^{8-10}$

In our study, only cdc2 and Ki67 are significantly correlated with the overall survival. The explanation for the lack of prognostic significance for $\alpha$-tubulin and CENP-F is unclear, but if further confirmed, raises the possibility of significant differences between the expression level of RNA and that of the protein for these markers. Since our assessment of expression was based on the percentage of cells reactive with the markers, rather than on total expression, it is also possible that the uneven distribution of $\alpha$-tubulin and CENP-F among neoplastic cells may lead to an 'underestimation' of their expression using immunohistochemistry. The other possibilities may include the use of different patient population and differences in treatment.

Importantly, the prognostic values of cdc2 and Ki67 are independent of IPI and clinical stage. Both IPI and clinical stage have been found to be prognostically important in some but not all studies of mantle cell lymphoma. ${ }^{2-5}$ In our study, no statistical significance was found with IPI except when the cutoff was lowered to 1 . As shown in Table 1 , there were only seven (23\%) patients in our study who have an IPI of $\leq 1$, which relatively limits the prognostic utility of IPI for most mantle cell lymphoma patients. Most patients in our study fell into Ann Arbor clinical stage III and IV and we found no statistical significance in survival between patients with stage III and IV. These findings support that immunodetection of cdc2 and Ki67 as a useful method in assessing the prognosis of mantle cell lymphoma patients. With the commercial availability of these antibodies, and the ease of applying these antibodies to formalin-fixed/paraf- 
fin-embedded tissues, we suggest the assessment of cdc2 and Ki67 expression to be included in the diagnostic work-up of new mantle cell lymphoma cases. In contrast to Ki67, cdc2 represents more than a proliferation marker. Its crucial role in cell cycle regulation suggests a mechanism for tumorigenesis in mantle cell lymphoma. The therapy of mantle cell lymphoma has advanced in recent years with the use of newer reagents such as rituximab and proteosome inhibitors. It is hoped that biologically rational advances in therapy will overcome the negative prognostic value of cdc2 and other markers in this disease. It is also of interest to assess the prognostic significance of cdc2 and other prognostic markers in mantle cell lymphoma patients treated with these new modalities.

In addition to prognostic indications, the identification of novel molecular markers may provide new targets for therapeutic intervention. Indeed, the use of flavopiridol in mantle cell lymphoma patients is based on the characteristic overexpression of cyclin D1 in this lymphoma type. Flavopiridol is a cyclin-dependent kinase modulator that induces cell cycle arrest via inhibition of cyclin-dependent kinase activities and reduction of cyclin D1 levels. ${ }^{28}$ Recently, a phase II study with flavopiridol reported limited success with only three out of 28 patients demonstrating partial response, and the rest having either stable disease or progressive disease. ${ }^{29}$ Determination of cdc2 level in these patient samples may help identify a subgroup of patients more susceptible to flavopiridol.

\section{Acknowledgements}

This study is partially supported by grants from the Alberta Cancer Board and the University of Alberta Hospital Foundation to RL. We thank Darryl Glubrecht for his assistance with immunohistochemistry.

\section{References}

1 Jaffe ES, Stein HN, Vardiman JW. Pathology and Genetics of Tumours of Haematopoietic and Lymphoid Tissues: WHO Classification of tumours. IARC Press: Lyon 2001.

2 Andersen NS, Jensen MK, de Nully Brown P, et al. A Danish population-based analysis of 105 mantle cell lymphoma patients: incidences, clinical features, response, survival and prognostic factors. Eur J Cancer 2002;38:401-408.

3 Weisenburger DD, Vose JM, Greiner TC, et al. Mantle cell lymphoma. A clinicopathologic study of 68 cases from the Nebraska Lymphoma Study Group. Am J Hematol 2000;64:190-196.

4 Raty R, Franssila K, Joensuu H, et al. Ki-67 expression level, histological subtype, and the International Prognostic Index as outcome predictors in mantle cell lymphoma. Eur J Haematol 2002;69:11-20.
5 Bernard M, Gressin R, Lefrere F, et al. Blastic variant of mantle cell lymphoma: a rare but highly aggressive subtype. Leukemia 2001;15:1785-1791.

6 Ek S, Bjorck E, Porwit-MacDonald A, et al. Increased expression of Ki-67 in mantle cell lymphoma is associated with de-regulation of several cell cycle regulatory components, as identified by global gene expression analysis. Haematologica 2004;89: 686-695.

7 Schrader C, Meusers P, Brittinger G, et al. Topoisomerase IIalpha expression in mantle cell lymphoma: a marker of cell proliferation and a prognostic factor for clinical outcome. Leukemia 2004;18:1200-1206.

8 Greiner TC, Moynihan MJ, Chan WC, et al. p53 mutations in mantle cell lymphoma are associated with variant cytology and predict a poor prognosis. Blood 1996;87:4302-4310.

9 Hernandez L, Fest T, Cazorla M, et al. p53 gene mutations and protein overexpression are associated with aggressive variants of mantle cell lymphomas. Blood 1996;87:3351-3359.

10 Louie DC, Offit K, Jaslow R, et al. p53 overexpression as a marker of poor prognosis in mantle cell lymphomas with t(11;14)(q13;q32). Blood 1995;86:2892-2899.

11 Chiarle R, Budel LM, Skolnik J, et al. Increased proteasome degradation of cyclin-dependent kinase inhibitor p27 is associated with a decreased overall survival in mantle cell lymphoma. Blood 2000;95: 619-626.

12 Bodrug SE, Warner BJ, Bath ML, et al. Cyclin D1 transgene impedes lymphocyte maturation and collaborates in lymphomagenesis with the myc gene. EMBO J 1994;13:2124-2130.

13 Martinez N, Camacho FI, Algara P, et al. The molecular signature of mantle cell lymphoma reveals multiple signals favoring cell survival. Cancer Res 2003;63: 8226-8232.

14 Rosenwald A, Wright G, Wiestner A, et al. The proliferation gene expression signature is a quantitative integrator of oncogenic events that predicts survival in mantle cell lymphoma. Cancer Cell 2003; 3:185-197.

15 Motulsky H. Comparing groups with confidence intervals. In: Intuitive Biostatistics. Oxford University Press: New York, 1995.

16 Bosch F, Lopez-Guillermo A, Campo E, et al. Mantle cell lymphoma: presenting features, response to therapy, and prognostic factors. Cancer 1998;82: 567-575.

17 Korkolopoulou P, Vassilakopoulos TP. Topoisomerase IIalpha as a prognostic factor in mantle cell lymphoma. Leukemia 2004;18:1347-1349.

18 Velders GA, Kluin-Nelemans JC, De Boer CJ, et al. Mantle-cell lymphoma: a population-based clinical study. J Clin Oncol 1996;14:1269-1274.

19 Hofmann WK, de Vos S, Tsukasaki K, et al. Altered apoptosis pathways in mantle cell lymphoma detected by oligonucleotide microarray. Blood 2001;98: 787-794.

20 Goodger NM, Gannon J, Hunt T, et al. The localization of p34cdc2 in the cells of normal, hyperplastic, and malignant epithelial and lymphoid tissues of the oral cavity. J Pathol 1996;178:422-428.

21 Ito Y, Yoshida H, Matsuzuka F, et al. Cdc2 expression in primary thyroid lymphoma: its relationship with biological aggressiveness and G2 cyclins. Pathol Res Pract 2003;199:533-538. 
22 Wolowiec D, Berger F, Ffrench P, et al. CDK1 and cyclin A expression is linked to cell proliferation and associated with prognosis in non-Hodgkin's lymphomas. Leuk Lymphoma 1999;35:147-157.

23 Zhu X, Chang KH, He D, et al. The C terminus of mitosin is essential for its nuclear localization, centromere/kinetochore targeting, and dimerization. J Biol Chem 1995;270:19545-19550.

24 Casiano CA, Landberg G, Ochs RL, et al. Autoantibodies to a novel cell cycle-regulated protein that accumulates in the nuclear matrix during $\mathrm{S}$ phase and is localized in the kinetochores and spindle midzone during mitosis. J Cell Sci 1993;106:1045-1056.

25 Landberg G, Erlanson M, Roos G, et al. Nuclear autoantigen p330d/CENP-F: a marker for cell proliferation in human malignancies. Cytometry 1996;25: 90-98.
26 Erlanson M, Casiano CA, Tan EM, et al. Immunohistochemical analysis of the proliferation associated nuclear antigen CENP-F in non-Hodgkin's lymphoma. Mod Pathol 1999;12:69-74.

27 Clark GM, Allred DC, Hilsenbeck SG, et al. Mitosin (a new proliferation marker) correlates with clinical outcome in node-negative breast cancer. Cancer Res 1997;57:5505-5508.

28 Senderowicz AM. Small-molecule cyclin-dependent kinase modulators. Oncogene 2003;22:66096620.

29 Kouroukis CT, Belch A, Crump M, et al. Flavopiridol in untreated or relapsed mantle-cell lymphoma: results of a phase II study of the National Cancer Institute of Canada Clinical Trials Group. J Clin Oncol 2003;21: 1740-1745. 